\title{
LOCAL SPECTRUM AND GENERALIZED SPECTRUM
}

\author{
MOSTAFA MBEKHTA
}

(Communicated by Palle E. T. Jorgensen)

\begin{abstract}
This paper provides the proofs of those results announced in $[5, \S 5]$ that deal with the connection between the regular set and the local resolvant set of closed operators on a Hilbert space. We also give some characterizations and properties of Cowen-Douglas operators.
\end{abstract}

\section{INTRODUCTION}

Let $H$ be a Hilbert space and let $A$ be a closed operator with domain $D(A)$ and range $R(A)$ in $H, N(A)$ and $\sigma(A)$ will respectively denote the kernel and the spectrum of $A$.

If $U$ is a subset of $\mathbb{C}$, we say that $A$ has a generalized resolvent operator $R g(A, \lambda)$ in $U$; if $\forall \lambda \in U, \operatorname{Rg}(A, \lambda)$ is a bounded operator of $H$ into $D(A)$ such that

$$
\begin{aligned}
(A-\lambda I) R g(A, \lambda)(A-\lambda I) & =(A-\lambda I) \\
\operatorname{Rg}(A, \lambda)(A-\lambda I) \operatorname{Rg}(A, \lambda) & =\operatorname{Rg}(A, \lambda)
\end{aligned}
$$

[4-6] contain discussions of a generalization of the resolvent set $\rho(A)$ (the complement of $\sigma(A)$ with respect to $\mathbb{C}$ ), where the notion of inverse operator is replaced by that of generalized inverse operator. Let $\operatorname{reg}(A)$ denote the regular set of $A$ defined by

$$
\begin{aligned}
\operatorname{reg}(A)=\{\lambda \in \mathbb{C} ; A \text { has a generalized resolvent, } & \\
& \text { analytic on a neighborhood } U \text { of } \lambda\} .
\end{aligned}
$$

It is clear that $\operatorname{reg}(A)$ is open and contains $\rho(A)$. The generalized spectrum of $A, \sigma_{g}(A)$ (the complement of $\operatorname{reg}(A)$ with respect to $\mathbb{C}$ ), has properties analogous to those of $\sigma(A)$ in classical spectral theory (cf. [5, 6]).

We shall say that $A$ is regular if $R(A)$ is closed and $\forall n>0, N\left(A^{n}\right) \subseteq R(A)$.

In [4, Theorem 2.6] it was shown that $\lambda \in \operatorname{reg}(A)$ if and only if $A-\lambda I$ is regular. The core of $A$, denoted by $\operatorname{Co}(A)$, is, by definition, the largest subspace $M$ of $H$ such that $A(M \cap D(A))=M . \operatorname{Co}(A-\lambda I)$ is constant on each connected component of $\operatorname{reg}(A)$. If $A$ is regular, then $\operatorname{Co}(A)$ is closed

Received by the editors October 27, 1989 and, in revised form, March 21, 1990.

1980 Mathematics Subject Classification (1985 Revision). Primary 47A05; Secondary 47B40.

Key words and phrases. Local spectrum, generalized spectrum, core of operator, Cowen-Doglas operators. 
and $\operatorname{Co}(A)=\bigcap_{n \geq 0} R\left(A^{n}\right)=\bigcap_{n \geq 0} R\left(A-\lambda_{n} I\right)$, where $\left\{\lambda_{n}\right\}$ is a sequence of two by two distinct points of the component of $\operatorname{reg}(A)$ that contains zero and is convergent to zero (cf. [4]).

If $u \in H$, denote by $\delta_{A}(u)$ the set of $\mu \in \mathbb{C}$ such that there exists a neighborhood $V_{\mu}$ of $\mu$ in $\mathbb{C}$ and a function $f: V_{\mu} \rightarrow D(A)$ analytic on $V_{\mu}$ such that

$$
\forall \lambda \in V_{\mu}, \quad(A-\lambda I) f(\lambda)=u .
$$

$\delta_{A}(u)$ is called the local resolvent set of $A$ at $u$ and its complement with respect to $\mathbb{C}$, denoted by $\gamma_{A}(u)$, is called the local spectrum of $A$ at $u$ (cf. [1, $7,8])$.

The first section of this paper provides the proofs of the results announced in $[5, \S 5]$, that deal with the connection between the regular set and the local resolvent set of closed operators on a Hilbert space. It should be observed that Corollary 1.3 is a local version of [1, Chapter I, Proposition 3.7] and that Corollary 1.12 is a generalization of that same proposition.

In $\S 2$ of this paper, we give some characterizations and properties of CowenDouglas operators (Theorem 2.5 and Corollary 2.6).

\section{RESUlts}

Theorem 1.1. Let $A$ be a closed operator; $G$ a connected component of $\operatorname{reg}(A)$; and $\lambda_{0} \in G$. Then

$$
G \subseteq \delta_{A}(u) \Leftrightarrow u \in \operatorname{Co}\left(A-\lambda_{0} I\right) .
$$

Proof. $(\Rightarrow) \lambda_{0} \in G \subseteq \delta_{A}(u)$. Then by definition, $\exists U_{0}$ a neighborhood of $\lambda_{0}$ in $\mathbb{C}$, and $f: U_{0} \rightarrow D(A)$ is a function analytic on $U_{0}$ such that $\forall \lambda \in U_{0}$, $(A-\lambda I) f(\lambda)=u$. Hence $\forall \lambda \in U_{0}, u \in R(A-\lambda I)$, and, in particular, $u \in \bigcap_{j>0} R\left(A-\lambda_{j} I\right)$, where $\left\{\lambda_{j}\right\}$ is a sequence of points of $G \cap U_{0}$ two by two distinct that converges to $\lambda_{0}$. Therefore $u \in \operatorname{Co}\left(A-\lambda_{0} I\right)$.

$(\Leftarrow)$ Let $\mu \in G$. By definition of $\operatorname{reg}(A), \exists U_{\mu} \subseteq G$ a neighborhood of $\mu$ in $\mathbb{C}$ and a generalized resolvent operator $R g(A, \lambda)$ of $A$ analytic on $U_{\mu}$. Furthermore $P_{\lambda}=(A-\lambda I) R g(A, \lambda)$ is a projection such that $R\left(P_{\lambda}\right)$ $=R(A-\lambda I) . \operatorname{Co}(A-\lambda I)$ is constant on $G$; hence, $\forall \lambda \in U_{\mu}, u \in \operatorname{Co}\left(A-\lambda_{0} I\right)=$ $\operatorname{Co}(A-\lambda I) \subseteq R(A-\lambda I)$, whence $\forall \lambda \in U_{\mu}, u=P_{\lambda} u$. Therefore $\forall \mu \in G, \exists U_{\mu}$ neighborhood of $\mu$ and $f(\lambda)=R g(A, \lambda) u$ analytic on $U_{\mu}$ such that $\forall \lambda \in U_{\mu}$, $(A-\lambda I) f(\lambda)=(A-\lambda I) R g(A, \lambda) u=P_{\lambda} u=u$, whence $\mu \in \delta_{A}(u)$, and therefore $G \subseteq \delta_{A}(u)$.

Corollary 1.2. Let $A$ be a closed operator, and let $\operatorname{reg}(A)=\bigcup_{i \geq 0} G_{i}$. Then, if $u \in H$,

$$
\operatorname{reg}(A) \subseteq \delta_{A}(u) \Leftrightarrow u \in \bigcap_{\lambda_{i} \in G_{i}} \operatorname{Co}\left(A-\lambda_{i} I\right) .
$$

Corollary 1.3. Let $A$ be a closed operator; $G$ a connected component of $\operatorname{reg}(A)$; and $u \in H$. Then

$$
G \cap \delta_{A}(u) \neq \varnothing \Rightarrow G \subseteq \delta_{A}(u)
$$


Proof. Let $\lambda_{0} \in G \cap \delta_{A}(u)$. Then $u \in \operatorname{Co}\left(A-\lambda_{0} I\right)$ (cf. [7, Proposition 1.3]). Using Theorem 1.1, it follows that $G \subseteq \delta_{A}(u)$.

Corollary 1.4. Let $A$ be a regular operator and $G$ be the connected component of $\operatorname{reg}(A)$ that contains zero. Consider the equation

$$
(A-\lambda I) x=y,
$$

where $y \in H$ and $\lambda \in G$. Then

(1) $y \in \operatorname{Co}(A)$ if and only if there exists a function $x(\lambda)$, having values in $H$ and analytic in $G$, that is a solution of $(\mathrm{E})$.

(2) If $y \notin \operatorname{Co}(A)$, (E) has a solution only for isolated $\lambda$ in $G$.

Proof. (1) is an immediate consequence of Theorem 1.1.

(2) Let $y \notin \operatorname{Co}(A)$; and suppose there exists a sequence $\left\{\lambda_{n}\right\}_{n>1}$ of two by two distinct points of $G$ that accumulate at a point $\lambda_{0} \in G$, and that (E) has a solution for each $\lambda_{n}$. Then $y \in \bigcap_{n \geq 1} R\left(A-\lambda_{n} I\right)=\operatorname{Co}\left(A-\lambda_{0} I\right)$, and since $\operatorname{Co}(A-\lambda I)$ is constant on $G$, it follows that $y \in \operatorname{Co}(A)$, a contradiction.

Remark 1.5. Set $H_{1}(A)=\bigcap_{\lambda_{i} \in G_{i}} \operatorname{Co}\left(A-\lambda_{i} I\right)$. Then the following inclusions are true:

$$
\rho(A) \subseteq \bigcap_{u \in H} \delta_{A}(u) \subseteq \operatorname{reg}(A) \subseteq \bigcap_{u \in H_{1}(A)} \delta_{A}(u) .
$$

Indeed, the middle inclusion follows from the fact,

$$
\bigcap_{u \in H} \delta_{A}(u) \subseteq\{\lambda \in \mathbb{C} ; R(A-\lambda I)=H\} \subseteq \operatorname{reg}(A) .
$$

The last inclusion follows from Corollary 1.2. It is a proper inclusion, as is shown by the following example:

Let $H=\ell^{2} ;\left\{e_{n}\right\}$ be its natural basis; and $A$ be the operator defined by $A e_{n}=e_{n+1}$. It is easy to see that $N(A)=\{0\}$ and that $R(A)$ is closed. Hence, $A$ is regular. Also $\operatorname{Co}(A)=\bigcap_{n \geq 0} R\left(A^{n}\right)=\{0\}$, hence $\left.H_{1}(A)=0\right\}$, and therefore $\bigcap_{u \in H_{1}(A)} \delta_{A}(u)=\mathbb{C}$. On the other hand, $\operatorname{reg}(A)=\mathbb{C} \backslash \Pi$ where $\Pi$ is the unit circle.

Lemma 1.6. Denote by $H_{0}(A)=\left\{u \in D^{\infty}(A) ; \lim _{n \rightarrow \infty}\left\|A^{n} u\right\|^{1 / n}=0\right\}$ the quasinilpotent part of $A$. Then

(i) $\forall n \geq 0, N\left(A^{n}\right) \subseteq H_{0}(A)$.

(ii) If $A$ is regular then, $\overline{H_{0}(A)}=\overline{\bigcup_{n \geq 0} N\left(A^{n}\right)} \subseteq \operatorname{Co}(A)$.

Proof. (i) Obvious. (ii) See [4, Proposition 2.10].

Proposition 1.7. Let $A$ be a densely defined closed operator on $H$ such that $A$ and $A^{*}$ have the S.V.E.P. (single value extension property). Then

$$
\rho(A)=\operatorname{reg}(A) \text {. }
$$

Proof. It suffices to show that $\operatorname{reg}(A) \subseteq \rho(A)$. From [7, Corollary 1.5] it follows that if $A$ has the S.V.E.P., then $\forall \lambda \in \operatorname{reg}(A), N(A-\lambda I)=\{0\}$. By symmetry 
between $A$ and $A^{*}$, we see that $\forall \lambda \in \operatorname{reg}(A), N\left(A^{*}-\bar{\lambda} I\right)=\{0\}$, and therefore $\operatorname{reg}(A) \subseteq \rho(A)$.

Corollary 1.8. Let $A$ be a densely defined closed operator on $H$ such that $A$ and $A^{*}$ have the S.V.E.P. Then

$A$ is semi-Fredholm $\Rightarrow A$ is a Riesz-Schauder operator.

Proof. If $A$ is semi-Fredholm, then there exists a neighborhood $U$ of the origin such that $\forall \lambda \in U \backslash\{0\}, A-\lambda I$ is regular (see [3, Proposition 4.3.1a]). According to the preceding proposition, it follows that $\forall \lambda \in U \backslash\{0\}, A-\lambda I$ is invertible. From the continuity of the index, we conclude that $A$ is a Fredholm operator with index zero.

Moreover, if $M$ and $N$ provide a Kato decomposition of degree $d$ of $A$, then $A_{\mid M}$ is regular and $A_{\mid N}$ is nilpotent of order $d$. Let us show that $M \cap H_{0}(A)=\operatorname{Co}(A) \cap H_{0}(A)$. Indeed $\operatorname{Co}(A) \subseteq M \Rightarrow \operatorname{Co}(A) \cap H_{0}(A) \subseteq$ $M \cap H_{0}(A)$. Furthermore, $M \cap H_{0}(A)=H_{0}\left(A_{\mid M}\right)$, and the fact that $A_{\mid M}$ is regular implies that $M \cap H_{0}(A) \subseteq \operatorname{Co}(A)$. Hence $M \cap H_{0}(A)=\operatorname{Co}(A) \cap H_{0}(A)$. But $A$ has the S.V.E.P., which implies that $\operatorname{Co}(A) \cap H_{0}(A)=\{0\}$ (cf. [7, Corollary 1.5]), and hence that $M \cap H_{0}(A)=\{0\}$.

On the other hand, $N \subseteq N\left(A^{d}\right) \subseteq H_{0}(A) \Rightarrow H_{0}(A)=M \cap H_{0}(A) \oplus N$. Therefore $H_{0}(A)=N \subseteq N\left(A^{d}\right)$, whence $\forall n \geq d, N\left(A^{n}\right) \subseteq N\left(A^{d}\right)$ so that $N\left(A^{d}\right)=N\left(A^{d+1}\right)$. Finally, by making use of the symmetry between $A$ and $A^{*}$, we see that $R\left(A^{d}\right)=R\left(A^{d+1}\right)$.

Proposition 1.9. Let $A$ be a regular operator and $M$ a closed subspace of $H$ invariant by $A$, such that $H_{0}(A) \subseteq M$. Then $A_{\mid M}$ is regular.

Proof. Let us show first that $R\left(A_{\mid M}\right)=A(M \cap D(A))$ is closed. Observe that

$$
N(A) \subseteq H_{0}(A) \subseteq M \Rightarrow M=N(A) \oplus M \cap N(A)^{\perp} .
$$

Let $u_{n} \in R\left(A_{\mid M}\right)$. Then $\exists v_{n} \in D(A) \cap M$ such that $u_{n}=A v_{n}$. It follows from (1) that the $v_{n} \perp N(A)$ can be chosen. Suppose that $u_{n} \rightarrow u$. Since $R(A)$ is closed, it follows that $v_{n} \rightarrow v$ and, since $M$ is closed, $v \in M$, whence $u=A v \in R\left(A_{\mid M}\right)$.

It remains to show that $\forall n \geq 0, N\left(\left(A_{\mid M}\right)^{n}\right) \subseteq R\left(A_{\mid M}\right)$. Since $A$ is regular, $\forall n \geq 0, N\left(A^{n}\right) \subseteq R(A)$, which implies that, if $u \in N\left(A^{n}\right)$, then $\exists v \in D(A)$ such that $u=A v \in H_{0}(A) \subseteq M$. Thus $u \in M$ and $v \in N\left(A^{n+1}\right) \subseteq M$, and therefore $\forall u \in N\left(A^{n}\right)$, we have $u \in R\left(A_{\mid M}\right)$. Since $N\left(\left(A_{\mid M}\right)^{n}\right) \subseteq N\left(A^{n}\right)$, it follows that $\forall n \geq 0, N\left(\left(A_{\mid M}\right)^{n}\right) \subseteq R\left(A_{\mid M}\right)$, and that $A_{\mid M}$ is regular.

Corollary 1.10. Let $A$ be a regular operator with $N(A)=\{0\}$. Then for every closed subspace $M$ of $H$ invariant by $A, A_{\mid M}$ is regular.

Proof. Using Lemma 1.6(ii), we see that $R(A)$ is closed and that the equation $N(A)=\{0\}$ implies that $H_{0}(A)=\{0\}$. Hence, for every subspace $M$ of $H$, $H_{0}(A) \subseteq M$, and the conclusion follows from the preceding proposition. 
Corollary 1.11. Let $A$ be a closed operator that has the S.V.E.P. Then, for every closed subspace of $H$ invariant by $A, \operatorname{reg}(A) \subseteq \operatorname{reg}\left(A_{\mid M}\right)$.

Proof. According to Proposition 1.7, if $A$ has the S.V.E.P., then $\forall \lambda \in \operatorname{reg}(A)$, $N(A-\lambda I)=\{0\}$. The rest of the proof is a consequence of the previous corollary.

Corollary 1.12. Let $A$ be a closed operator that has the S.V.E.P., and let $G$ be a connected component of $\operatorname{reg}(A)$. Then for every closed subspace of $H$ invariant by $A$,

$$
G \cap \sigma\left(A_{\mid M}\right) \neq \varnothing \Rightarrow G \subseteq \sigma\left(A_{\mid M}\right) .
$$

Proof. According to Corollary 1.11, $G$ is contained in a component of $\operatorname{reg}\left(A_{\mid M}\right)$. Moreover, $A$ has the S.V.E.P.. Then $\sigma\left(A_{\mid M}\right)=\bigcup_{u \in M} \gamma_{A_{\mid M}}(u)$. Now the proof follows from applying Corollary 1.3 to the operator $A_{\mid M}$.

Remark. This last result is a generalization of [1, Chapter I, Proposition 3.7].

\section{APPLiCATIONS to COWEN-DOUglas OPERATORS}

The following lemmas will be needed:

Lemma 2.1. Let $A$ be a closed and regular operator with $D(A)$ dense on $H$. Then

$$
\operatorname{Co}\left(A^{*}\right)=H_{0}(A)^{\perp} \text {. }
$$

Proof. $A$ is regular and $D(A)$ is dense on $H$, implying that $A^{*}$ is regular and $\forall j \geq 0, N(A j)^{\perp}=R\left(A^{* j}\right)$ (see [4, Proposition 2.8]). Let $A_{1}^{*}: \operatorname{Co}\left(A^{*}\right) \rightarrow$ $\operatorname{Co}\left(A^{*}\right)$, the restriction of $A^{*}$ to $\operatorname{Co}\left(A^{*}\right)$. Then $A_{1}^{*}$ is onto, and there is a bound operator $B$ of $\operatorname{Co}\left(A^{*}\right)$ that takes its values in $\operatorname{Co}\left(A^{*}\right) \cap D\left(A^{*}\right)$ such that $\forall u \in \operatorname{Co}\left(A^{*}\right), A_{1}^{*} B u=u$. Consequently, $\forall u \in \operatorname{Co}\left(A^{*}\right), \exists v_{n}=B^{n} u$ such that $u=A^{* n} v_{n}$ and $\left\|v_{n}\right\| \leq\|B\|^{n}\|u\|$ for all $n \geq 0$.

Let $u \in \operatorname{Co}\left(A^{*}\right)$ and $w \in H_{0}(A)$. Then $(w, u)=\left(w, A^{*_{n}} v_{n}\right)=\left(A^{n} w, v_{n}\right)$. Therefore, $\forall n \geq 0,|(w, u)| \leq\left\|A^{n} w\right\|\left\|v_{n}\right\| \leq\|B\|^{n}\left\|A^{n} w\right\|\|u\|$. Since $w \in$ $H_{0}(A)$, then $\lim _{n \rightarrow \infty}\|B\|^{n}\left\|A^{n} w\right\|=0$. Therefore, $(w, u)=0$. This shows that $H_{0}(A) \subseteq \operatorname{Co}\left(A^{*}\right)^{\perp}$ or $\operatorname{Co}\left(A^{*}\right) \subseteq H_{0}(A)^{\perp}$.

Inversely, $\forall j \geq 0, N\left(A^{j}\right) \subseteq H_{0}(A)$ whence $\forall j \geq 0, H_{0}(A)^{\perp} \subseteq N\left(A^{j}\right)^{\perp}=$ $R\left(A^{*_{j}}\right)$. Therefore $H_{0}(A)^{\perp} \subseteq \bigcap_{j \geq 0} R\left(A^{*_{j}}\right)=\mathrm{Co}\left(A^{*}\right)$.

Lemma 2.2. Let $A$ be a closed and regular operator with $D(A)$ dense on $H$. Then the map $\lambda \mapsto \overline{H_{0}(A-\lambda I)}$ is constant on each connected component of $\operatorname{reg}(A)$.

Proof. The proof of this lemma is a consequence of Lemma 2.1 and the fact that the core is constant within every connected component of $\operatorname{reg}(A)$.

Lemma 2.3. Let $A$ be a closed and regular operator with $D(A)$ dense on $H$ and $\Omega$ a connected component of $\operatorname{reg}(A)$ that contains zero. Then

$$
\overline{H_{0}(A)}=\text { c.l.m. }\{N(A-\lambda I)\}_{\lambda \in \Omega}=\text { c.l.m. }\left\{N\left(A^{n}\right)\right\}_{n \geq 0},
$$

where c.l.m. denotes closed linear manifold. 
Proof. Observe that $\forall \lambda \in \mathbb{C}, N(A-\lambda I) \subseteq H_{0}(A-\lambda I)$. Using Lemma 2.2, we deduce that $\forall \lambda \in \Omega, N(A-\lambda I) \subseteq \overline{H_{0}(A)}$. Consequently c.l.m. $\{N(A-\lambda I)\}_{\lambda \in \Omega} \subseteq$ $\overline{H_{0}(A)}$.

Inversely, let $u \in$ [c.l.m. $\left.\{N(A-\lambda I)\}_{\lambda \in \Omega}\right]^{\perp}$, particularly $u \in N\left(A-\lambda_{i} I\right)^{\perp}$ where $\left\{\lambda_{i}\right\}_{i>0}$ is a sequence of two by two distinct points of $\Omega$ and is convergent to zero. Therefore $u \in R\left(A^{*}-\bar{\lambda}_{i} I\right)$, which implies $u \in \bigcap_{i>0} R\left(A^{*}-\bar{\lambda}_{i} I\right)$ $=\operatorname{Co}\left(A^{*}\right)$. Hence [c.l.m. $\left.\{N(A-\lambda I)\}_{\lambda \in \Omega}\right]^{\perp} \subseteq \operatorname{Co}\left(A^{*}\right)=H_{0}(A)^{\perp}$, and therefore $\overline{H_{0}(A)} \subseteq$ c.l.m. $\{N(A-\lambda I)\}_{\lambda \in \Omega}$.

See [4, Proposition 2.10] for the proof of the second equality in this lemma.

Definition 2.4 (see [2]). We say that $T \in B(H)$ is a Cowen-Douglas operator if there exist a connected open set $\Omega$ of $\mathbb{C}$ and a positive integer $n$ such that

(1) $\Omega \subseteq \sigma(T)$.

(2) $R(T-\omega I)=H, \forall \omega \in \Omega$.

(3) $\operatorname{dim} N(T-\omega I)=n, \forall \omega \in \Omega$.

(4) c.l.m. $\{N(T-\omega I)\}_{\omega \in \Omega}=H$.

In this case, we shall denote $T \in B_{n}(\Omega)$.

Remark. (2) and (4) $\Rightarrow$ (1); indeed, (2) $\Rightarrow \Omega \subseteq \operatorname{reg}(T)$ and (4) $\Rightarrow$ $H_{0}(T-\omega I)=H$. Therefore $N(T-\omega I) \neq\{0\}$ (see Lemma 2.3); consequently, $\Omega \subseteq \sigma(T)$.

Theorem 2.5. Let $T \in B(H)$. Then the following conditions are equivalent:

(i) there exists a connected open set $\Omega$ of $\mathbb{C}$ containing zero and a positive integer $n$, such that $T \in B_{n}(\Omega)$.

(ii) $\operatorname{Co}(T)=H, \overline{H_{0}(T)}=H$, and $\operatorname{dim} N(T)=n$.

(iii) $\operatorname{Co}(T)=H, \operatorname{Co}\left(T^{*}\right)=\{0\}$, and $\operatorname{dim} N(T)=n$.

(iv) $T^{*}$ regular, $\operatorname{Co}\left(T^{*}\right)=\{0\}$, and $\operatorname{codim} R\left(T^{*}\right)=n$.

Proof. (i) $\Rightarrow$ (ii) is an immediate consequence of Lemma 2.3 and Definition 2.4 .

(ii) $\Rightarrow$ (iii) is a consequence of Lemma 2.1 .

(iii) $\Rightarrow$ (iv) is obvious.

For (iv) $\Rightarrow$ (i), note that $T^{*}$ regular $\Rightarrow N\left(T^{*}\right) \subseteq \operatorname{Co}\left(T^{*}\right)=\{0\}$ and $R\left(T^{*}\right)$ closed. It is easy to see that $R(T)=H$ and that $\operatorname{Co}(T)=H$. Since $\operatorname{Co}(T)=$ $H, T$ is regular and there exists a neighborhood $\Omega$ of the origin such that $\forall \omega \in \Omega, T-\omega I$ is regular, and $R(T-\omega I)=H$. The rest of the proof is a consequence of Lemma 2.1, Lemma 2.3, and [6, Theorem 2.1].

Corollary 2.6. Let $T \in B_{n}(\Omega)$. Then

(1) $\sigma(T)$ is connected,

(2) $\sigma_{w}(T)=\sigma(T)$,

(3) $\sigma_{p}\left(T^{*}\right)=\varnothing$,

(4) $\operatorname{ind}(T-\lambda I) \geq 0, \forall \lambda \in \rho_{S-F}(T)$,

(5) $T^{*}$ has the S.V.E.P. and $T$ does not have the S.V.E.P. at all $\omega \in \Omega$, 
where $\rho_{S-F}(T)=\{\lambda \in \mathbb{C} ; T-\lambda I$ is semi-Fredholm $\}$ and $\sigma_{w}(T)=\{\lambda \in \mathbb{C} ;$ $T-\lambda I$ is not semi-Fredholm of index 0$\}$, is the Weyl spectrum of $T$.

Proof. Without loss of generality, we can assume that $0 \in \Omega$. (1), (2), and (3) are deduced from $[6, \S 2]$ and Theorem 2.5, since $\operatorname{Co}\left(T^{*}\right)=\{0\}$ and $N\left(T^{*}\right)=$ $\{0\}$.

(4) is a consequence of (3).

(5) $\sigma_{p}\left(T^{*}\right)=\varnothing \Rightarrow T^{*}$ has the S.V.E.P.. Moreover, if $T$ has the S.V.E.P. in $\Omega$, then Proposition 1.7 implies $\Omega \subseteq \rho(T)$.

Corollary 2.7. Let $\Omega$ be a connected open set of $\mathbb{C}$ and $n$ a positive integer. Then $B_{n}(\Omega) \cap\{T \in B(H) ; T$ is decomposable $\}=\varnothing$.

Proof. From Theorem 2.5, $T \in B_{n}(\Omega) \Rightarrow \mathrm{Co}\left(T^{*}\right)=\{0\}$. On the other hand, the fact that $T$ is decomposable implies that $T^{*}$ is decomposable. Using [7, Corollary 2.2(ii)], we deduce that $T^{*}$ is quasinilpotent; therefore, $\sigma(T)=\{0\}$, which is a contradiction, because $\Omega \subseteq \sigma(T)$.

\section{REFERENCES}

1. I. Colojoara and C. Foias, Theory of generalized spectral operators, Gordon and Breach, New York, 1968.

2. M. J. Cowen and R. G. Douglas, Complex geometry and operator theory, Acta. Math. 141 (1978), 187-261.

3. J. P. Labrousse, Les opérateurs quasi-Fredholm: une généralisation des opérateurs semiFredholm, Rend. Circ. Math. Palermo Ser. II. 29 (1980), 161-258.

4. M. Mbekhta, Généralisation de la décomposition de Kato aux opérateurs paranormaux et spectraux, Glasgow Math. J. 29 (1987), 159-175.

5. __ Sur la théorie spectrale généralisée, C. R. Acad. Sci. Paris 306 (1988), 593-596.

6. __ Résolvant généralisé et théorie spectrale, J. Operator Theory 21 (1989), 69-105.

7. _ Théorie spectrale locale et limite de nilpotents, Proc. Amer. Math. Soc. (to appear).

8. F. H. Vasilescu, Analytic functional calculus and spectral decompositions, D. Reidel, 1982.

Université de Lille I, U.F.R. Mathématiques, 59655 Villeneuve d’AscQ CedeX 